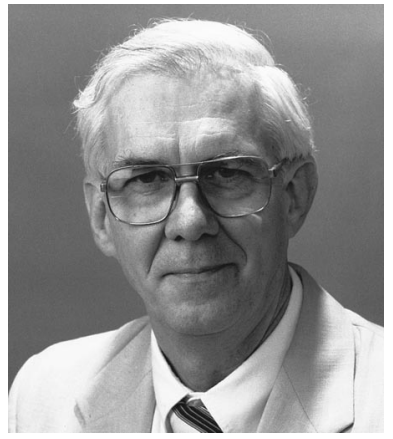

\section{SPIE with/and/or OSA?}

By now I would hope that most members of SPIE and OSA have read the messages by Roland Jacobsson and M. J. Soileau, President and Past-President of SPIE, and Gary Bjorklund, President of OSA. For those who have not, the messages can be found on the SPIE and OSA home pages at www.spie.org and www.osa.org, respectively. Both letters make a case for closer collaboration with the other organization and both give a list of projects that the two societies have collaborated on recently. I urge you to read them.

Anyone who has spent any time working on the governance of these two societies quickly becomes aware of the differences between these organizations. The differences are not in the operation of the societies-all nonprofit professional organizations operate in pretty much the same way. They publish, organize conferences, and run exhibitions for the benefit of their members. It is the approaches that the membership and leadership take to the governance of their societies that distinguish them.

Let me state the images of the two societies with the contrast set to maximum and displayed in 2-bit mode.

-SPIE is run for a bunch of low-life optical engineers who publish unrefereed papers in yellow books and attend conferences filled with speakers recruited from the four ends of the earth to fill a program that you wouldn't send your graduate student to.

-OSA is an organization of esthete academic optical scientists who publish out-of-date papers so esoteric that only three other people in the universe can read them (two of whom are working in the author's lab) in order to obtain the luxurious life of a tenured professor and permit travel to exotic meetings whose content is tightly controlled by a small circle of old boys.

There! Did I get it right?

If, however, we turn down the contrast and increase the number of gray levels, the images contain lots more detail and variety. But the rude truth in the black and white images is that SPIE and OSA are two different cultures. This is born out in the fact that only $7 \%$ of the combined membership are members of both societies. If there are distinctly different cultures in these two societies, why do anything? Or to put it in the lingo of South Georgia: "If it ain't broke, don't fix it!'

One reason that Gary Bjorklund mentioned in his letter to OSA members was that:

"In many cases, meetings compete for the limited time and resources of people working in the community. The single most common member complaint I hear is: 'There are too many competing meetings ....',

While I can understand the difficulty, since I am the SPIE Symposia Chair this year, this alone is not a compelling reason for some type of closer strategic alliance that could range anywhere from joint committees to federation to merger.

The SPIE Presidents' letter mentions the international aspect of the collaboration:

"This [collaboration with other societies] is an essential mode of operation for an international society like SPIE because it is vitally important to support and work closely with national or regional organizations while striving to meet SPIE's mission to serve the global optics and photonics community. This is not only professional courtesy, it also makes good business sense because it avoids duplication and combines scarce resources and organizational strengths in pursuing common objectives.'

Yet, even this is not a compelling reason to work toward unity between the two societies. As I look at the operations of the two societies as a member of both and as editor of this journal, I cannot point to a single issue that is so divisive that it threatens either or both the two societies. So why contemplate increased collaboration? We can just shake hands and continue to spar with each other when scheduling meetings, publishing optics texts and journals, and competing in the international arena. Friendly competition keeps everyone sharp. But we would be missing something: a great many opportunities to do more, do it better, and use resources more effectively and efficiently. There is inherent strength, and synergism, in unity. 
To me, increased efforts at collaboration should not be directed at just solving problems. There are regular meetings of SPIE and OSA officers to handle them. Instead, these efforts should be directed at creating opportunities for members that do not exist yet. The world is changing rather quickly these days. Consider... how would the conduct of your professional life change if you lost your email for a month? (Yes, yes, I know there are times you would like it to go away altogether!) Optics science and engineering rests on a ground of rapid and constant communication, both formal and informal, and the ground has been shifting.

Recently, I was informed (by e-mail) that:

"As an SPIE Member, you may supplement your print subscription with an e-journal(s) for $\$ 25$ per journal, or convert your print subscription to an online subscription. The site uses PDF (Portable Document Format) and includes these features:

-Browsable HTML table of contents with a link to PDF display of the full article.

-Searchable database of complete bibliographic record for each article (authors, titles, keywords, abstracts) with a link to the full article.

-Full article viewing, searching, and printing using Adobe Acrobat Reader.

-Articles available online about two weeks before the print version is published.',

Two days later I was notified by OSA that, as a subscriber to Optics Letters, I could add Optics Letters Online for a small fee. Again, it details much the same features, noting that "Optics Letters Online enhances your subscription to Optics Letters by providing more timely dissemination of the latest research as well as search capabilities and reference linking not possible with the print version.",

Wow! The researcher's dream: access to most of the papers he or she needs at the push of a button. Well, almost. It turns out that you have to be either a subscriber to the journal to be able to open the document beyond the abstract or else pay a fee to have the document downloaded. Much of the ability to link is illusory. Only if the documents are formatted properly will it be possible to achieve hyperlinked nirvana. But what if the integration between OSA and SPIE was such that they could publish a fully integrated digital database of literature: journals, books, TOPS, and even those ubiquitous conference proceedings. The ability to provide hotlinks between referenced publications would be tremendous help to anyone trying to understand current work in the field. This goes beyond "ease of use." It is, if you will, "ease of information." This will be nearly impossible without some integration of publishing operations. Considering the large impact that publications have on the financial operations of both societies, it is hard to see how it could be done without a close alliance with common objectives.

The other opportunity that a strategic alliance between the societies would bring is visibility. In interferometry, it is just another name for contrast. But in the world of public policy, it tends to be a function of reputation and size. Considering the growing influence of optics in technology and commerce, it is a pity that the representation of the optics community is split between two very good organizations. This occurs at a time when our field is beginning to emerge as one comparable in influence to the "traditional', fields (electrical engineering, chemistry, mechanical engineering, etc.). For example, the job of optical scientist or optical engineer does not merit a separate category in a national description of intellectual resources. So it comes down to the question of who "speaks" for optics and who will provide the new tools for professional advancement in the field? OSA? SPIE? The answer should be: Both-together.
Donald C. O'Shea Editor 